\title{
Association of endocrine active environmental compounds with body mass index and weight loss following bariatric surgery
}

Harshal Deshmukh ${ }^{1 *}$, Lesa L Aylward ${ }^{2 *}$, Martin Rose ${ }^{3}$, Alwyn Fernandes ${ }^{4}$, Peter Sedman ${ }^{1}$, Natalie J Thatcher ${ }^{5}$, Stephen L Atkin ${ }^{6}$, Thozhukat Sathyapalan ${ }^{1}$

1)Hull York Medical School, University of Hull, United Kingdom 2) Summit Toxicology, LLP, Falls Church, VA, USA 3) University of Manchester 4) School of Environmental Sciences, University of East Anglia, Norwich, UK 5) European Food Safety Authority, Parma, Italy 6) Royal College of Surgeons in Ireland, Bahrain

*Joint first authors

Source of funding: This study was supported by the Food Standards Agency, United Kingdom (T01063). The sponsors did not influence the study design; the collection, analysis, and interpretation of data; the writing of the report; and the decision to submit the paper for publication. Any views or opinions expressed are solely those of the authors and do not necessarily represent those of the FSA.

Author LLA provides consulting services to a wide variety of clients on chemical risk assessment, including regarding chemicals included in this study. The remaining authors have no relevant financial interests to disclose.

Conflict of interest

I certify that neither I nor my co-authors have a conflict of interest as described in the disclosure policy of Endocrine Society that is relevant to the subject matter or materials included in this Work.

\section{Corresponding author}

Dr Harshal Deshmukh MBBS MRCP MPH PhD

Clinical Lecturer in Endocrinology and Diabetes

Academic Diabetes, Endocrinology and Metabolism

University of Hull and Hull York Medical School

220 - 236 Anlaby Road

Hull, HU3 2RW, UK

Tel: +441482675312

Email: harshaldeshmukh@,nhs.net

This is the peer reviewed version of the following article: Deshmukh, H., Aylward, L.L., Rose, M., Fernandes, A., Sedman, P., Thatcher, N.J., Atkin, S.L. and Sathyapalan, T. (2020), Association of endocrine active environmental compounds with body mass index and weight loss following bariatric surgery. Clin Endocrinol., which has been published in final form at https://doi.org/10.1111/cen.14257. This article may be used for non-commercial purposes in accordance With Wiley Terms and Conditions for self-archiving. 


\begin{abstract}
Introduction

The objective of this study was to study associations of a wide range of halogenated biphenyls, dibenzo-p-dioxins, dibenzofurans, and diphenylethers with body mass index (BMI) and evaluate changes in their concentration following bariatric surgery.
\end{abstract}

\title{
Methods
}

Subcutaneous fat, visceral fat, and liver tissue samples were collected from 106 patients undergoing Roux-en-Y gastric bypass surgery for weight loss or patients who were undergoing abdominal surgery for non-bariatric reasons. We measured concentrations of an extensive panel of chlorinated and brominated biphenyls, dioxins, and furans, and brominated diphenylethers in the samples. We conducted linear regression to examine associations with BMI, adjusting for age and gender. Changes in concentration for indicator chemicals were evaluated in samples collected following bariatric surgery in a small sub-population.

\section{Results}

After adjustments for age and gender and correction for multiple testing seven ortho-chlorinated biphenyls, one non-ortho-chlorinated biphenyl, four PCDD/F's and one ortho-brominated biphenyl were associated with BMI. The strongest associations between BMI and lipid-adjusted concentrations were seen with PCB-105 in subcutaneous fat (beta=16.838 P-val=1.45E-06) PCB-126 in visceral fat (beta=15.067 P-val=7.72E-06) and PCB-118 (beta=14.101 P-val=2.66E-05) in liver. The concentrations of sum PCBs, chlorinated TEQ's and brominated compounds increased significantly with weight loss in subcutaneous fat in a group of ten individuals resampled up to five years after bariatric surgery and substantial weight loss.

\section{Conclusion}


We show that selected polychlorinated biphenyls PCBs and structurally related polychlorinated dibenzo-p-dioxins dibenzofurans ( $\mathrm{PCDD} / \mathrm{Fs})$ were associated with BMI. Concentrations of these lipophilic compounds in subcutaneous fat increased following bariatric surgery. 


\section{Introduction}

Halogenated biphenyls, dibenzo-p-dioxins, dibenzofurans, and diphenylethers are chemicals that can interfere with endocrine systems and are implicated in the pathogenesis of obesity, cancer, birth defects, and other developmental disorders(1-5). There are increasing concerns that some of these chemicals can interfere with regulatory processes involved in lipid metabolism and in the control of adipocyte function resulting in excess weight and obesity $(6$, 7). While some of these chemicals are of natural origin, the majority are synthetic chemicals, which have been released by human activities into the environment. Among the chemicals in this broad category are polychlorinated biphenyls (PCBs) and structurally related polychlorinated dibenzo-p-dioxins and dibenzofurans ( $\mathrm{PCDD} / \mathrm{Fs})$, the brominated dioxins (PBDDs), furans (PBDFs), and biphenyls (PBBs), diphenyl ethers (PBDEs)(8, 9). The patterns of distribution of these chemicals in the body have been studied in numerous species(10). These chemicals are highly lipophilic and tend to distribute in the body primarily on the basis of the lipid content of the tissue, with data indicating that in general, the concentration of these compounds in lipid throughout the body is usually very similar(8). $\mathrm{PCDD} / \mathrm{Fs}, \mathrm{PBDD} / \mathrm{Fs}$, and coplanar PCBs are also found in liver tissue as they bind to a specific protein, cytochrome p4501a2, which is present in the liver and can be induced by dioxin activation of the aryl hydrocarbon receptor $(11,12)$. As a result, hepatic concentrations of these compounds can significantly exceed the concentrations expected on the basis of the lipid content of the liver.

Concentrations of many of these environmental chemicals have been monitored in the blood in the US population through the US National Health and Nutrition Examination Survey (NHANES), however, to our knowledge, there have been no systematic biomonitoring 
studies for chlorinated dioxins in UK populations and there are no UK studies looking at the association of environmental chemicals with BMI and obesity. Furthermore, since the concentrations of these compounds vary with the lipid content in the body, there are concerns that their concentrations might increase in the body following rapid weight lipid loss following procedures such as bariatric surgery(13-15). There are limited data looking at the changes in the body concentrations of these environmental chemicals following bariatric surgery and no data looking at the changes of these in liver tissue following weight $\operatorname{loss}(16$, 17). For example, Jansen A et.al.(18) analyzed 17 different polyfluoroalkylated substances (PFASs) to assess the effect of weight loss before and one year after bariatric surgery, and showed that the the plasma levels of all PFASs decreased upto 4-34\%. Another study(19) examined 17 persistent organic pollutants (POPs) and 13 PFAA at baseline and following bariatric surgery and showed that there was a significant increase in POP levels in response obesity surgery.

The prevalence of obesity is increasing in the western world and genetic factors explain only a small proportion of the heritability of $\mathrm{BMI} / \mathrm{obesity}(20,21)$. It is therefore important to investigate the association of known potentially endocrine active compounds with BMI. The objectives of this study were 1) to look at the association of selected persistent lipophilic environmental chemicals with BMI and 2) compare the concentration of these compounds in the body before and after bariatric surgery in a subset of the study population.

\section{Methods}

\section{Study population and sample collection}


The study population consisted of patients undergoing Roux-en-Y gastric bypass surgery for weight loss or patients who were undergoing abdominal surgery for non-bariatric reasons (mainly Nissen fundoplication for gastro-oesophageal reflux disease) from April 2010 until March 2015. Informed consent for the study was obtained and National Research Ethics Committee, Yorkshire and Humber - South Yorkshire, UK (REC reference 10/H1304/13), approved the study. Anthropometric parameters including height and weight were measured on the day of surgery. During surgery, tissue samples were taken including liver $(500 \mathrm{mg})$ and adipose tissue (visceral and subcutaneous: $40 \mathrm{~g}$ each). Opportunistic contacts for follow-up sampling with the participants were also made when they attended the bariatric surgery follow-up clinic. For those who consented, subcutaneous fat biopsies were taken at follow-up. An incision was made in the anterior abdominal wall under local anaesthesia after sterile precaution and subcutaneous fat biopsies were taken. Samples were stored at $-80^{\circ} \mathrm{C}$ until packed in dry ice for transport to the UK National Reference Laboratory for Contaminants in Food and Feed (FERA) laboratory for analysis. After receipt at the FERA laboratory, samples were stored at $-20^{\circ} \mathrm{C}$ until analysis. These dioxins are considered very stable compounds and are highly persistent in vivo and vitro with an estimated half life of 9-15 yrs on soil surfaces(22).

\section{Sample analysis}

The method (23) used for the preparation, extraction and analysis of samples is comprehensively validated, formally accredited and forms part of the modular CEN method EN16215:2012. In brief, samples were fortified with 13C-labelled analogues of target compounds and exhaustively extracted using mixed organic solvents. PBDEs and ortho-substituted $\mathrm{PCBs} / \mathrm{PBBs}$ were separated from non-ortho substituted $\mathrm{PCBs} / \mathrm{PBBs}$, 
PCDD/Fs and PBDD/Fs by fractionation on activated carbon. The two fractions were further purified using adsorption chromatography on alumina. Analytical measurement was carried out using high-resolution gas chromatography-high resolution mass spectrometry (HRGC-HRMS) for all analytes apart from the ortho-substituted PCBs which were analysed by high-resolution gas chromatography-unit resolution mass spectrometry (HRGC-LRMS). Lipid content was measured using a method based on BS:4401: Part 41970 (Werner-Schmidt Method).

The analysis is accredited (UKAS) to ISO 17025 standards, with the inclusion of an in-house reference material and method blanks which were evaluated prior to reporting of sample data and used to determine the limits of detection. Further quality assurance measures included the successful participation in international inter-comparison exercises such as Dioxins in Food-2011 to 2014, and EURL-run PT exercises on dioxins, dioxin-like PCBs, ICES-6 PCBs and PBDEs. Additionally, quality control evaluation for the accompanying data follows the criteria specified for chlorinated dioxins and PCBs (Commission Regulation 252/2012, (European Commission, 2012). Not every targeted sample was collected, and for a variety of reasons, the number of analysed samples varied by matrix and chemical compound group. For example, in some cases, particularly for liver samples, the collected sample was of insufficient mass to allow analysis for all of the target chemical groups. In some cases the analytical results were flagged as "indicative" and were inconsistent with other data collected for the same compounds, and the decision was made to exclude those analytical results from the statistical analyses. Finally, for brominated dioxins and furans, 17 out of 21 contributing to toxic equivalency (TEQ) were measured due to the lack of available standards. Specifically, octa-brominated dioxin and furan were not measured and only one 
hepta-brominated (a hepta-brominated furan) was measured. Although these have low toxic equivalency factor (TEF) values they are likely to contribute to total brominated TEQ, so it is likely that the brominated TEQ values will be somewhat underestimated.

\section{Statistical methods}

Summary statistics for each measured congener in each tissue (detection frequency, geometric mean, geometric standard deviation, minimum and maximum) were generated in $\mathrm{R}$ 3.5.0.

Several studies have shown a significant association of age with distribution of dioxin chemicals in the population. A simple bivariate correlation which will be confounded by age, is therefore not useful and can be misleading. Hence, we assessed the association of these environmental chemicals using regression analysis adjusting for both age and gender. Linear regression analysis implemented in $\mathrm{R}$ was used to test the association of BMI with the $\log 10$-transformed concentration of each compound, adjusted for age and gender. Bonferroni correction was used to identify a threshold for statistical significance $(0.05 /$ number of tests $)$ to account for multiple testing. Since the environmental chemicals showed a high degree of correlation in subcutaneous tissues, visceral fat and liver we used a conservative Bonferroni correction of $0.05 / 63$ where 63 is the number of chemicals measured across all the tissues.

Chlorinated PCBs and PCDD/Fs were analysed in baseline and follow-up samples from 10 participants. Sensitivity analysis was performed by 1) imputing missing data with random forest algorithm imputation in $\mathrm{R}$ and 2) by using raw values on environmental chemicals (rather than log-transformed). 
To assess changes in analyte concentrations following weight loss, changes in the sum of three persistent indicator PCBs $(138,153$, and 180) and in lower bound estimates of chlorinated ED were examined. Baseline and follow-up fat samples were analysed for selected brominated compounds in 10 individuals. BDE 153 was selected as a marker to examine changes in persistent brominated compound concentrations in these two participants. Shapiro-Wilk tests were used to compare the median concentrations of the analytes before and after the bariatric surgery.

\section{Results}

Supplementary Figure 1 shows the study flow chart. Table 1 shows the demographic characteristics of the study populations and the compound measured in the study. The study consisted of 106 participants with 64\% females with a median age of 55 (IQR $=44-56$ ) years and a median $\mathrm{BMI}$ of $37(\mathrm{IQR}=27-37) \mathrm{kg} / \mathrm{m}^{2}$. We looked at eighty-one environmental chemicals consisting of twenty-one ortho-chlorinated biphenyls, four non-ortho-chlorinated biphenyls, seventeen PCDD/Fs, seventeen polybrominated diphenyl ethers, seven ortho-brominated biphenyls, 3 non-ortho-brominated biphenyls and 12 PBDD/Fs. Of the 81 compounds, 18 were not included in subsequent analyses because detection frequencies were below $65 \%$ in all tissues. Supplementary table 1 and $\mathbf{2}$ shows the summary statistics for the concentrations of each analyte in subcutaneous fat, visceral fat and liver. Concentrations of most of these chemicals show high correlation with each other and are also highly correlated in subcutaneous fat, visceral fat and liver (Supplementary figures 1, 2, 3, 4).

Figure 1 shows a heatmap of the beta coefficients for associations between BMI and $\log _{10}$-transformed, lipid-adjusted concentrations of 63 compounds after adjustments for age 
and gender in subcutaneous fat, visceral fat and liver. Highly significant associations between BMI and compounds are indicated with darker shades of red while less significant associations are shown in blue. After adjustments for age and gender and correction for multiple testing ( $\mathrm{P}<0.007$, the threshold for Bonferroni correction) BMI was significantly associated with seven ortho-chlorinated biphenyls, one non- ortho-chlorinated biphenyl, four PCDD/F's and one ortho-brominated biphenyl (Table 2). The strongest associations were seen for PCB-105 in subcutaneous fat (beta=16.838 P-val=1.45E-06) PCB-126 in visceral fat (beta=15.067 P-val=7.72E-06) and PCB-118 (beta=14.101 P-val=2.66E-05) in liver. All associations were positive except for 2,2',4,4',5,5'-hexabromobiphenyl (BB-153)in the liver, which showed a negative correlation with BMI.

\section{Changes in the concentrations selected compounds following a bariatric surgery}

Follow-up subcutaneous fat biopsies of sufficient volume to allow analysis were collected from 10 bariatric surgery group participants. The biopsies were collected following an average of 2.8 years after initial surgery (range 1 to 4.8 years) and participants lost an average of $35.3 \%$ of their pre-surgery body weight. Fat mass was estimated at the baseline surgery time point and at the follow-up sample time using a prediction formula based on age, gender, and $\mathrm{BMI}(24)$. Based on these estimates, the participants lost an average of $48.6 \%$ of their body fat (range 38.1 to $60.6 \%$ ). Figure $2 \mathbf{a}$ and $2 \mathbf{b}$ shows the concentrations of a sum of key indicator PCBs $(138,153$, and 180) and chlorinated toxic equivalency (TEQ) before and after bariatric surgery. In the 10 follow-up participants, the mean concentration of the sum of indicator PCBs increased from $111.74 \mu \mathrm{g} / \mathrm{kg}$ lipid to $240.6 \mu \mathrm{g} / \mathrm{kg}(\mathrm{P}=0.02)$ while the mean chlorinated TEQ concentration increased from $10.6 \mathrm{ng} / \mathrm{kg}$ lipid to $23.0 \mathrm{ng} / \mathrm{kg}$ lipid $(\mathrm{P}=0.02)$. 
BDE-153 was also measured in two participants before and after surgery and the mean concentrations increased $2.5 \mu \mathrm{g} / \mathrm{kg}$ lipid to $4.04 \mu \mathrm{g} / \mathrm{kg}$ lipid.

\section{Discussion}

This is the first study in the UK population looking at the association of a suite of lipophilic, persistent chlorinated and brominated biphenyls, dioxins, furans, and diphenylethers with BMI as well as changes in their concentration following bariatric surgery and weight loss. We show that several ortho-chlorinated biphenyls and non-ortho-chlorinated biphenyls and PCDD/F's are associated with BMI and that the concentrations of these environmental chemicals in fat tissues increase following bariatric surgery.

Our study showed that BMI was positively associated with selected polychlorinated biphenyls PCBs and structurally related polychlorinated dibenzo-p-dioxins dibenzofurans (PCDD/Fs) whereas BMI was not generally associated with the brominated dioxins (PBDDs), furans (PBDFs), and biphenyls (PBBs), diphenyl ethers (PBDEs) after correction for multiple testing. The positive association between concentrations of many of these compounds and age has been well documented in the literature; however, studies looking at the association with BMI with environmental chemicals have been equivocal(25). Since the increase in BMI also correlates with an increase in body lipids the concentrations of environmental chemicals in the body can increase or decrease depending upon the time course of environmental exposure to these compounds, acquisition of body fat, and lipophilic properties of these compounds. As a result, it is difficult to establish a causal relationship between these 
compounds and BMI. A study by Dirinck et al.(26) showed negative correlation between BMI, waist, fat mass percentage, total and subcutaneous abdominal adipose tissue, and serum levels of PCB 153, 180, 170, and the sum PCBs but positive association of $\beta$-hexachlorocyclohexane $(\beta \mathrm{HCH})$ with BMI. On the other hand studies have also indicated that increasing body mass index (BMI) may be associated with increased serum lipid concentrations of dioxins (Collins et al. 2007; Flesch-Janys et al. 1996; Garabrant et al. 2009; Kim et al. 2011; Landi et al. 1998; Michalek et al. 1996; Ott et al. 1993, Tuomisto et al. Toxicol Lett. 2016). Many of these studies have been conducted in occupationally exposed populations who have elevated serum concentrations of PCDD/Fs compared to the general population. Relatively little information is available to assess the impact of obesity on background chlorinated dioxin levels, and no information is available for brominated dioxins. With the increasing prevalence of obesity in the general population in many countries, evaluation of if, and how much, dioxin levels are associated with obesity becomes relevant to risk assessment for these compounds. There are various sources of these endocrine active chemicals, and according to some studies, exposure to chlorinated POPs by the general population continues, mostly through consumption of fatty foods of animal origin. Thus a high intake of animal source food can lead to higher levels of endocrine active chemicals leading to higher BMI.

The associations between BMI and environmental chemicals during the developmental period and childhood can be explored by prospective cohort studies looking at the exposure to environmental chemicals at birth and follow-up weight in the study participants; however, such studies have been limited. In a Belgian prospective cohort study(27) in the paediatric population, cord blood PCB (congeners 118, 138, 153, 170, and 180) concentrations were 
associated with increased follow-up BMI. Another study(28) showed that female foetuses with the highest transplacental PCB exposures were heavier for their heights than other girls (at age 11 years) who were not exposed, however, these associations were only significant in caucasian participants. However, other studies have shown either negative(29) or null associations between PCBs and BMI(30).

Animal studies have also provided some evidence showing associations with obesity. A study by Ruzzin et al.(31) showed that male rats fed a high-fat diet of crude salmon oil (HFC) containing high levels of persistent organic pollutants (POPs) or refined salmon oil (HFR) with significantly lower environmental chemicals levels showed increased abdominal obesity, and elevated levels of diacylglycerol, triacylglycerol, and total cholesterol in HFC rats compared with the HFR rats. HFC-fed rats developed insulin resistance, impaired lipid and glucose metabolism, and hepatosteatosis, confirming that chronic exposure to environmental chemicals severely impairs insulin sensitivity and contributes to abdominal obesity in male rats.

A further issue relates to the impact of weight reduction upon the concentration and amount of dioxins in the body. There is the possibility that significant weight reduction could cause release of dioxin from adipose tissue depots, as the adipose tissue is lost, and that the concentrations in blood and adipose tissue of dioxin and other toxic lipophilic chemicals could increase if the dioxin remains in the body as adipose tissue is lost, potentially increasing the internal exposure level for toxicologically relevant tissues or receptors. There is evidence that weight loss is associated with an increase in PCBs and other highly lipophilic contaminants (Charlier et al. 2002; Chevrier et al. 2000; Kim et al. 2011; Pelletier et al. 2002). However, the factors affecting the degree of increase in concentration, time profile of 
changes after weight loss, and other mass balance issues have not been thoroughly assessed to date. The benefits of acute weight loss would be the primary finding but in the long term when weight loss has plateaued then hypothetically the increased concentrations of these chemicals could have a detrimental effect, though longer studies are needed to confirm this.

In this study, we also show that estimated concentrations of selected compounds increased in remaining fat depots following weight loss induced by bariatric surgery. Our findings are in agreement with an earlier study $(16,32)$ which showed that the serum PCB concentrations increased by approximately $50 \%$ following weight loss at six months follow-up. The data presented in our study are generally consistent with the hypothesis that compounds stored in fat are mobilized and redistributed into remaining fat tissue following weight loss. There are reports of association of polychlorinated biphenyls (PCBs), . In our study the degree of change in observed concentrations of environmental chemicals following weight loss differed substantially by the individual, even taking into account the different amount of weight loss and estimated losses in fat mass. However, for each individual, the degree of change in concentration was very similar across contaminants, suggesting a consistent quantity of release of compound and redeposition into the remaining fat mass regardless of the specific contaminant. This observation suggests that the differing degrees of change in concentration may be related to interindividual differences in the accuracy of the fat mass estimation procedure used. Other possible explanations include a individual differences in elimination half-lives and by a small sample size of the follow-up.

Our study had several limitations. Since this is a cross-sectional study, we are not able to establish a causal link between environmental chemicals and BMI. It is possible that the association of the environmental chemicals with BMI simply reflects the excess accumulation 
of lipophilic environmental chemicals in participants with high BMI and high concentration of body lipids. Furthermore, in this cross-sectional study, we are not able to adjust for the timecourse of lifelong environmental exposure to these chemicals, which are important determinants of their concentration in the body. Also, the scope of this study was limited to investigating the association of these endocrine-active chemicals with BMI and changes in their concentration following bariatric surgery. Further studies are needed to investigate if the accumulation of these chemicals following bariatric surgery increases the likelihood of endocrine or hormonal dysfunction in the long term, though in the short term the beneficial effects of bariatric surgery would be more evident. All the participants from this study were recruited, from Hull and East Riding of Yorkshire, which is a highly stable population epidemiologically; therefore, different environmental exposure is less likely than in a mobile population, but a detailed migratory history was not obtained to confirm this.

However, this study provides first paired liver and adipose tissue samples from adults for a range of environmental chemicals, including some with no previous human liver tissue data. We also show that if an environmental chemical is associated with BMI, this association is likely to be consistent across subcutaneous fat, visceral fat and in liver fat and likely is a consequence of high correlation levels across these depots. We also provide evidence of environmental chemicals re-distribution following weight loss, which has implications for patients experiencing significant weight loss, whether following surgery, dietary restriction, or secondary to illness. In summary, we show an association between BMI and various polychlorinated biphenyls PCBs and structurally related polychlorinated dibenzo-p-dioxins dibenzofurans (PCDD/Fs) and increased subcutaneous fat concentrations following weight 
loss. Further molecular, genetic and animal studies are needed to elucidate potential causal associations between these compounds and obesity..

\section{Data availability Statement}

The data that support the findings of this study are available from the corresponding author uponreasonable request. 
Table 1: Demographic characteristics of the study population and chemicals measured in the study

\begin{tabular}{|c|c|}
\hline Age & $55(\mathrm{IQR}=44-56)$ \\
\hline Gender \%females & $64 \%$ \\
\hline BMI & $37(\mathrm{IQR}=27-37)$ \\
\hline Ortho-chlorinated biphenyls & $\begin{array}{c}\text { PCB 18, PCB 28, PCB 31,PCB 47, PCB 49.PCB 51, PCB 52, PCB 99, PCB 101, PCB 105, PCB 114, PCB } 11 \\
157, \text { PCB 167, PCB 180, PCB } 189\end{array}$ \\
\hline $\begin{array}{l}\text { Non-ortho-chlorinated } \\
\text { biphenyls }\end{array}$ & PCB 77, РCB 81,РCB 126, РCB 169 \\
\hline $\mathrm{PCDD} / \mathrm{Fs}$ & $\begin{array}{l}\text { 2,3,7,8-TCDD; 1,2,3,7,8-PeCDD; 1,2,3,4,7,8-HxCDD; 1,2,3,6,7,8-HxCDD; 1,2,3,7,8,9-HxCDD; 1,2,3,4,6 } \\
\text { 2,3,4,7,8-PeCDF; 1,2,3,4,7,8-HxCDF ; 1,2,3,6,7,8-HxCDF ; 1,2,3,7,8,9-HxCDF ; 2,3,4,6,7,8-HxCDF ; } 1\end{array}$ \\
\hline $\begin{array}{l}\text { Polybrominated diphenyl } \\
\text { ethers }\end{array}$ & BDE 17,BDE 28,BDE 47,BDE 49,BDE 66,BDE 71,BDE 77,BDE 85,BDE 99,BDE 100,BDE 119,BDE \\
\hline $\begin{array}{l}\text { Ortho and non-ortho } \\
\text { brominated biphenyls }\end{array}$ & BB 15, BB 49,BB 52,BB 80,BB 101,BB 153,BB 209 BB 77,BB \\
\hline $\mathrm{PBDD} / \mathrm{Fs}$ & $\begin{array}{r}\text { 237-TriBDD,2378-TetraBDD, 12378-PentaBDD,123478/123678-HexaBDD, 123789-HexaBDD,238-TriBDF,23 } \\
\text { xaBDF,1234678-HeptabromoBDF }\end{array}$ \\
\hline
\end{tabular}


Table 2: Association of BMI with log-10 transformed concentrations (ng/g lipid, except where indicated) of various chlorinated and brominated compounds, adjusted for age and gender. Results reported only for associations significant with $\mathrm{P}<0.007$, the threshold for significance after Bonferroni correction in any of the three depots.

\begin{tabular}{|c|c|c|c|c|c|c|c|c|c|}
\hline \multirow[t]{2}{*}{ ind } & \multicolumn{3}{|l|}{ SCF } & \multicolumn{3}{|c|}{ Visceral Fat } & \multicolumn{3}{|l|}{ Liver } \\
\hline & Beta & $\mathrm{SE}$ & P-value & Beta & $\mathrm{SE}$ & P-value & Beta & SE & $\overline{\text { P-val }}$ \\
\hline & 13.28 & 4.16 & $2.23 \mathrm{E}-03$ & 13.04 & 4.24 & $2.99 \mathrm{E}-03$ & 12.39 & 4.11 & $3.81 \mathrm{E}$ \\
\hline & -11.56 & 6.4 & 0.07 & -17.59 & 5.35 & $1.55 \mathrm{E}-03$ & -17.59 & 5.35 & $\overline{0.001}$ \\
\hline & 11.09 & 4.40 & 0.01 & 11.33 & 4.55 & 0.01 & 11.47 & 3.80 & $3.81 \mathrm{E}$ \\
\hline & 10.5 & 3.27 & $2.13 \mathrm{E}-03$ & 10.79 & 3.37 & $2.02 \mathrm{E}-03$ & 3.98 & 3.36 & 0.24 \\
\hline $\mathrm{pg} / \mathrm{g}$ & 13.90 & 2.89 & $1.13 \mathrm{E}-05$ & 15.06 & 3.08 & $7.72 \mathrm{E}-06$ & 10.53 & 3.36 & $2.90 \mathrm{E}$ \\
\hline & 14.46 & 3.41 & $7.68 \mathrm{E}-05$ & 13.58 & 3.39 & $1.50 \mathrm{E}-04$ & 14.10 & 3.09 & $2.66 \mathrm{E}$ \\
\hline & 15.76 & 3.30 & $1.15 \mathrm{E}-05$ & 16.36 & 3.58 & $1.99 \mathrm{E}-05$ & 14.10 & 3.09 & $2.66 \mathrm{E}$ \\
\hline & 16.83 & 3.16 & $1.45 \mathrm{E}-06$ & 16.65 & 3.48 & $8.80 \mathrm{E}-06$ & 14.27 & 3.34 & $7.41 \mathrm{E}$ \\
\hline & -13.59 & 5.73 & 0.02 & 7.66 & 4.70 & 0.10 & -15.87 & 5.56 & $6.53 \mathrm{E}$ \\
\hline pg/g lipid & 13.01 & 4.45 & $4.93 \mathrm{E}-03$ & 14.85 & 4.56 & $1.84 \mathrm{E}-03$ & 2.90 & 4.98 & 056 \\
\hline xcdd, & 14.25 & 3.82 & $4.39 \mathrm{E}-04$ & 15.96 & 3.88 & $1.19 \mathrm{E}-04$ & 1.88 & 2.86 & 051 \\
\hline ixcdd, & 10.70 & 3.61 & $4.40 \mathrm{E}-03$ & 11.58 & 3.88 & $4.12 \mathrm{E}-03$ & $\mathrm{NC}$ & $\mathrm{NC}$ & $\mathrm{NC}$ \\
\hline hpcdd, & 9.05 & 2.79 & $1.99 \mathrm{E}-03$ & 9.47 & 3.10 & $3.32 \mathrm{E}-03$ & 5.14 & 3.63 & 0.16 \\
\hline
\end{tabular}

$\mathrm{NC}$ : not calculated due to low detection frequencies. Association of BMI with log-transformed environmental chemicals 


\section{References}

1. Kassotis CD, Stapleton HM. Endocrine-Mediated Mechanisms of Metabolic Disruption and New Approaches to Examine the Public Health Threat. Front Endocrinol (Lausanne). 2019;10:39.

2. Lauretta R, Sansone A, Sansone M, Romanelli F, Appetecchia M. Endocrine Disrupting Chemicals: Effects on Endocrine Glands. Front Endocrinol (Lausanne). 2019;10:178.

3. Terry MB, Michels KB, Brody JG, Byrne C, Chen S, Jerry DJ, et al. Environmental exposures during windows of susceptibility for breast cancer: a framework for prevention research. Breast Cancer Res. 2019;21(1):96.

4. Lymperi S, Giwercman A. Endocrine disruptors and testicular function. Metabolism. 2018;86:79-90.

5. Raghavan R, Romano ME, Karagas MR, Penna FJ. Pharmacologic and Environmental Endocrine Disruptors in the Pathogenesis of Hypospadias: a Review. Curr Environ Health Rep. 2018;5(4):499-511.

6. Soto AM, Sonnenschein C. Environmental causes of cancer: endocrine disruptors as carcinogens. Nat Rev Endocrinol. 2010;6(7):363-70.

7. Petrakis D, Vassilopoulou L, Mamoulakis C, Psycharakis C, Anifantaki A, Sifakis S, et al. Endocrine Disruptors Leading to Obesity and Related Diseases. Int J Environ Res Public Health. 2017;14(10).

8. van den Berg M, Denison MS, Birnbaum LS, Devito MJ, Fiedler $H$, Falandysz J, et al. Polybrominated dibenzo-p-dioxins, dibenzofurans, and biphenyls: inclusion in the toxicity equivalency factor concept for dioxin-like compounds. Toxicol Sci. 2013;133(2):197-208.

9. Van den Berg $M$, Birnbaum L, Bosveld AT, Brunstrom B, Cook P, Feeley $M$, et al. Toxic equivalency factors (TEFs) for PCBs, PCDDs, PCDFs for humans and wildlife. Environ Health Perspect. 1998;106(12):775-92.

10. Van den Berg M, De Jongh J, Poiger H, Olson JR. The toxicokinetics and metabolism of polychlorinated dibenzo-p-dioxins (PCDDs) and dibenzofurans (PCDFs) and their relevance for toxicity. Crit Rev Toxicol. 1994;24(1):1-74.

11. Diliberto JJ, Burgin D, Birnbaum LS. Role of CYP1A2 in hepatic sequestration of dioxin: studies using CYP1A2 knock-out mice. Biochem Biophys Res Commun. 1997;236(2):431-3.

12. DeVito MJ, Diliberto JJ, Ross DG, Menache MG, Birnbaum LS. Dose-response relationships for polyhalogenated dioxins and dibenzofurans following subchronic treatment in mice. I. CYP1A1 and CYP1A2 enzyme activity in liver, lung, and skin. Toxicol Appl Pharmacol. 1997;147(2):267-80.

13. Charlier C, Desaive C, Plomteux G. Human exposure to endocrine disrupters: consequences of gastroplasty on plasma concentration of toxic pollutants. Int J Obes Relat Metab Disord. 2002;26(11):1465-8.

14. Kim MJ, Marchand P, Henegar C, Antignac JP, Alili R, Poitou C, et al. Fate and complex pathogenic effects of dioxins and polychlorinated biphenyls in obese subjects before and after drastic weight loss. Environmental health perspectives. 2011;119(3):377-83.

15. Chevrier J, Dewailly E, Ayotte P, Mauriege P, Despres JP, Tremblay A. Body weight loss increases plasma and adipose tissue concentrations of potentially toxic pollutants in obese individuals. Int J Obes Relat Metab Disord. 2000;24(10):1272-8.

16. Dirinck E, Dirtu AC, Jorens PG, Malarvannan G, Covaci A, Van Gaal LF. Pivotal Role for the Visceral Fat Compartment in the Release of Persistent Organic Pollutants During Weight Loss. J Clin Endocrinol Metab. 2015;100(12):4463-71. 
17. Dirinck EL, Dirtu AC, Govindan M, Covaci A, Jorens PG, Van Gaal LF. Endocrine-disrupting polychlorinated biphenyls in metabolically healthy and unhealthy obese subjects before and after weight loss: difference at the start but not at the finish. Am J Clin Nutr. 2016;103(4):989-98.

18. Jansen A, Muller MHB, Gronnestad R, Klungsoyr O, Polder A, Skjerve E, et al. Decreased plasma levels of perfluoroalkylated substances one year after bariatric surgery. Sci Total Environ. 2019;657:863-70.

19. Rantakokko P, Mannisto V, Airaksinen R, Koponen J, Viluksela M, Kiviranta H, et al. Persistent organic pollutants and non-alcoholic fatty liver disease in morbidly obese patients: a cohort study. Environ Health. 2015;14:79.

20. Elks CE, den Hoed M, Zhao JH, Sharp SJ, Wareham NJ, Loos RJ, et al. Variability in the heritability of body mass index: a systematic review and meta-regression. Front Endocrinol (Lausanne). 2012;3:29.

21. Feng R. How much do we know about the heritability of BMI? Am J Clin Nutr. 2016;104(2):243-4.

22. Paustenbach DJ, Wenning RJ, Lau V, Harrington NW, Rennix DK, Parsons AH. Recent developments on the hazards posed by 2,3,7,8-tetrachlorodibenzo-p-dioxin in soil: implications for setting risk-based cleanup levels at residential and industrial sites. J Toxicol Environ Health. 1992;36(2):103-49.

23. Fernandes A, White S, D'Silva K, Rose M. Simultaneous determination of PCDDs, PCDFs, PCBs and PBDEs in food. Talanta. 2004;63(5):1147-55.

24. Deurenberg P, Weststrate JA, Seidell JC. Body mass index as a measure of body fatness: ageand sex-specific prediction formulas. Br J Nutr. 1991;65(2):105-14.

25. Malarvannan G, Dirinck E, Dirtu AC, Pereira-Fernandes A, Neels $H$, Jorens PG, et al. Distribution of persistent organic pollutants in two different fat compartments from obese individuals. Environ Int. 2013;55:33-42.

26. Dirinck E, Jorens PG, Covaci A, Geens T, Roosens L, Neels H, et al. Obesity and persistent organic pollutants: possible obesogenic effect of organochlorine pesticides and polychlorinated biphenyls. Obesity (Silver Spring). 2011;19(4):709-14.

27. Verhulst SL, Nelen V, Hond ED, Koppen G, Beunckens C, Vael C, et al. Intrauterine exposure to environmental pollutants and body mass index during the first 3 years of life. Environ Health Perspect. 2009;117(1):122-6.

28. Gladen BC, Ragan NB, Rogan WJ. Pubertal growth and development and prenatal and lactational exposure to polychlorinated biphenyls and dichlorodiphenyl dichloroethene. J Pediatr. 2000;136(4):490-6.

29. Blanck HM, Marcus M, Rubin C, Tolbert PE, Hertzberg VS, Henderson AK, et al. Growth in girls exposed in utero and postnatally to polybrominated biphenyls and polychlorinated biphenyls. Epidemiology. 2002;13(2):205-10.

30. Karmaus W, Osuch JR, Eneli I, Mudd LM, Zhang J, Mikucki D, et al. Maternal levels of dichlorodiphenyl-dichloroethylene (DDE) may increase weight and body mass index in adult female offspring. Occup Environ Med. 2009;66(3):143-9.

31. Ruzzin J, Petersen R, Meugnier E, Madsen L, Lock EJ, Lillefosse H, et al. Persistent organic pollutant exposure leads to insulin resistance syndrome. Environ Health Perspect. 2010;118(4):465-71.

32. Tuomisto J, Airaksinen R, Kiviranta H, Tukiainen E, Pekkanen J, Tuomisto JT. A pharmacokinetic analysis and dietary information are necessary to confirm or reject the hypothesis on persistent organic pollutants causing type 2 diabetes. Toxicology letters. 2016;261:41-8. 